\title{
BMJ Open Cohort profile: The Observational cohort for the study of DOlutegravir in Antiretroviral Combination REgimens (ODOACRE)
}

\author{
Arturo Ciccullo, ${ }^{1}$ Gianmaria Baldin (D , ${ }^{1}$ Amedeo Capetti, ${ }^{2}$ Vanni Borghi, ${ }^{3}$ \\ Gaetana Sterrantino, ${ }^{4}$ Alessandra Latini, ${ }^{5}$ Giordano Madeddu, ${ }^{6}$ Luigi Celani, ${ }^{7}$ \\ Francesca Vignale, ${ }^{8}$ Barbara Rossetti, ${ }^{9}$ Alex Dusina, ${ }^{1}$ Maria Vittoria Cossu, ${ }^{2}$ \\ Sibilla Restelli, ${ }^{2}$ William Gennari, ${ }^{3}$ Filippo Lagi, ${ }^{4}$ Andrea Giacomelli (D) , ${ }^{2}$ \\ Manuela Colafigli, ${ }^{5}$ Lucia Brescini, ${ }^{10}$ Alberto Borghetti, ${ }^{11}$ Cristina Mussini, ${ }^{3}$ \\ Stefano Rusconi (D) ,2 Simona Di Giambenedetto ${ }^{1,11}$
}

To cite: Ciccullo A, Baldin G, Capetti A, et al. Cohort profile: The Observational cohort for the study of DOlutegravir in Antiretroviral Combination REgimens (ODOACRE). BMJ Open 2019;9:e029960. doi:10.1136/ bmjopen-2019-029960

- Prepublication history for this paper is available online. To view these files, please visit the journal online (http://dx.doi org/10.1136/bmjopen-2019029960).

Received 19 February 2019 Revised 23 October 2019 Accepted 07 November 2019

Check for updates

(c) Author(s) (or their employer(s)) 2019. Re-use permitted under CC BY-NC. No commercial re-use. See rights and permissions. Published by BMJ.

For numbered affiliations see end of article.

Correspondence to Dr Gianmaria Baldin; gian.baldin@gmail.com

\section{ABSTRACT}

Purpose The Observational cohort for the study of DOlutegravir in Antiretroviral Combination REgimens (ODOACRE) cohort was established in Italy in 2016 to evaluate the overall efficacy and tolerability of dolutegravir (DTG)-based antiretroviral (ARV) regimens in clinical practice.

Participants The ODOACRE cohort enrols all adult HIV1-infected patients, both treatment-naïve and treatmentexperienced, starting a DTG-based ARV regimen, in 11 clinical centres in Italy from 2014.

Findings to date In recent years, various works by the ODOACRE cohort have been produced, demonstrating the high efficacy and tolerability of DTG-based ARV regimens in clinical practice, both in ART-naïve (in the setting of acute HIV-1 infection and late presenters patient) and experienced patients. We confirmed the virological efficacy of DTG-based regimens and we evaluated predictors of virological failure. We investigated cause of discontinuation and evaluated tolerability and metabolic profile of the regimens. Within these investigations, we explored particularly the use of DTG in simplification in two-drug regimen with either rilpivirine or lamivudine. We also compared DTG-based regimens with other integrase inhibitors in clinical practice.

Future plans To continue to study long-term efficacy and tolerability of DTG-based regimens is the purpose of the ODOACRE cohort.

\section{INTRODUCTION}

Since the introduction of combination antiretroviral therapy (cART) in the mid1990s, the management of HIV infection has dramatically changed along with the natural history and the prognosis of the disease. ${ }^{1}$ For decades, the standard of cART has been the association of two nucleos $(\mathrm{t})$ ide reverse transcriptase inhibitors $(\mathrm{N}(\mathrm{t})$ RTIs) with an anchor drug, namely, either a ritonavir-boosted protease inhibitor $(\mathrm{PI} / \mathrm{r})$
Strengths and limitations of this study

- The ODOACRE cohort includes 4139 patients, 481 of which are treatment naïve, who started a dolutegravir (DTG)-based regimen in each of the participating centres, providing a real clinical practice scenario of the use of DTG in Italy.

- Another strength is the cumulative length of followup (6937 patient-years of follow-up),which, along with the clinical, laboratory and demographic data of such a large number of patients, allows us to thoroughly define the safety and tolerability of the regimens.

- A predominant feature of our cohort is that it is prevalently composed of highly treatment experienced patients.

- The main weakness of our study is that it only collects routine clinical data and therefore some data could be missing in the analysis.

- Although a yearly update is planned, a delay exists between local data collection and availability of an updated central database.

or a non-nucleoside reverse transcriptase inhibitor (NNRTI). Such standard three-drug regimen proved to be effective in the vast majority of cases, obtaining and stably maintaining virological suppression and reducing the morbidity and mortality of HIV/AIDS. However, as longer follow-up data became available, clinician's attention shifted towards the toxicity of these aforementioned classes of antiretroviral (ARV) agents, with events such as osteoporosis and dyslipidaemia becoming increasingly evident in clinical practice. ${ }^{23}$ The marketing of the first-generation integrase inhibitors (INIs) represented a new turning point, which gave clinicians a new class of 


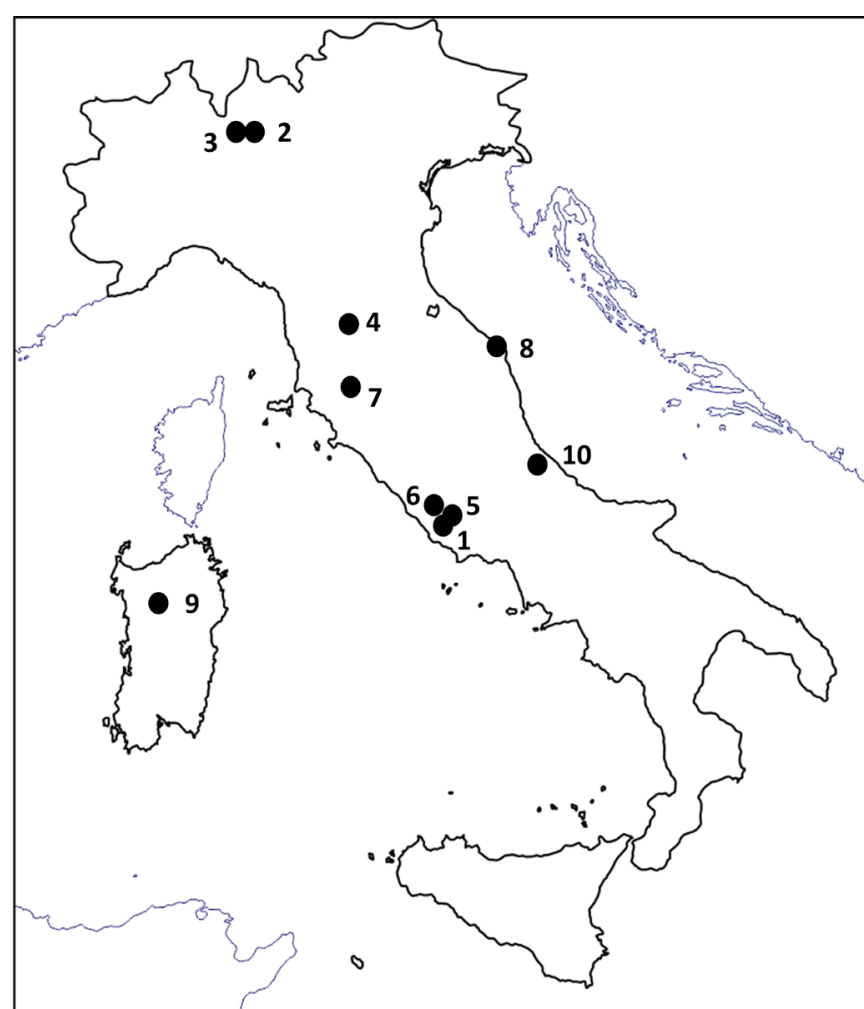

Figure 1 Enrolling centres of the ODOACRE cohort. (1) Infectious Diseases Unit, Catholic University of the Sacred Heart, Fondazione Policlinico Universitario Agostino Gemelli IRCCS, Rome. (2). Infectious Diseases Unit, DIBIC Luigi Sacco, University of Milan, Milan. (3) Division of Infectious Diseases, Department of Infectious Diseases, Luigi Sacco University Hospital, Milan. (4) Division of Tropical and Infectious Diseases, 'Careggi' Hospital, Florence. (5) Infectious Dermatology and Allergology Unit, IFO S. Gallicano Institute (IRCCS), Rome. (6) Department of Public Health and Infectious Diseases, Sapienza University of Rome, Rome. (7) University Division of Infectious Diseases, Siena University Hospital, Siena. (8) Clinic of Infectious Diseases, Azienda O.U. Ospedali Riuniti di Ancona, Ancona. (9) Department of Clinical and Experimental Medicine, University of Sassari, Sassari. (10) Infectious Disease Clinic, Department of Medicine and Science of Ageing, 'SS Annunziata' Hospital, Chieti. (11) Clinic of Infectious Diseases, Azienda Ospedaliero-Universitaria Policlinico, Modena.

molecules with a better tolerability profile compared with NNRTIs and PIs. The down sides were a low genetic barrier, the bis-in-die formulation of raltegravir (RAL) and the need for a pharmacological booster for elvitegravir (EVG), which hindered a vast use of these drugs as switch strategies in experienced patients. ${ }^{4}$

Dolutegravir (DTG), a second-generation INI, presented high efficacy in early clinical trials, along with minimal drug-drug interaction risk and overall favourable tolerability profile. ${ }^{5-7}$ DTG also presented a higher genetic barrier compared with first-generation $\mathrm{INI}^{8-10}$ and has become, since its introduction, one of the preferred choices by clinicians for both treatmentnaïve and experienced patients. ${ }^{11}{ }^{12}$ However, in recent years, observational studies showed conflicting data on DTG tolerability, with some cohorts reporting high rate of discontinuations of DTG-based regimens due to neuropsychiatric events. ${ }^{13}{ }^{14}$ DTG has also been related to neural-tube defects in newborns of mothers in treatment with this drug. ${ }^{15}$ Moreover, concerns have been raised about weight gain ${ }^{16}$ and a decline in renal function in patients taking DTG,${ }^{17}$ the latter a problem that could be related to the effect of DTG on organic cation transporter 2 (OCT2) ${ }^{18}$ To further investigate DTG tolerability and to assess the efficacy of DTG-based in clinical practice, several Italian clinicians joined their efforts creating a large observational cohort analysing patients starting DTG in real-life scenario.

\section{COHORT DESCRIPTION}

The Observational cohort for the study of DOlutegravir in Antiretroviral Combination REgimens (ODOACRE) was established in Italy in 2016 to evaluate the overall efficacy and tolerability of DTG-based regimens in clinical practice. Initially, the cohort was composed of 6 centres but rapidly expanded; currently, the ODOACRE cohort is composed of 11 centres, representing some of the most populated areas of the country (figure 1). In Italy, DTGbased regimens has become one of the first-line regimens both in ARV-naive patients and in treatment-experienced ones; moreover, DTG-based dual regimens are listed as possible switch strategies for both patients on standard three-drug regimen with a 2 NRTI-backbone, in order to reduce the risk of drug-related toxicity ('NRTI-sparing' approach) and in patients on PI-based or NNRTI-based strategies, in order to improve the metabolic profile and to reduce the risk of drug-drug interactions. ${ }^{19}$ The main objectives of the cohort are to assess the virological efficacy of DTG in real clinical practice settings and to assess the overall tolerability of DTG-based regimens. Moreover, the ongoing widespread use of less-drug regimens, following the results from clinical trials, showed promising results of DTG-based two-drug regimen both in treatment-experienced patients and in treatment-naïve subject. ${ }^{20-23}$ This fact has lead the ODOACRE cohort to focus on assessing the efficacy, safety and tolerability of DTG-based dual regimens (ie, DTG plus either rilpivirine, RPV, or lamivudine, 3TC) in clinical practice.

The ODOACRE cohort enrols all adult HIV-1infected patients, both treatment-naïve and treatmentexperienced, starting a DTG-based antiretroviral regimen in one of the enrolling centres. All patients signed informed consent for data collection. Children under 18 years of age and adults incapable of providing informed consent are excluded. At first visit, subjects are required to provide written informed consent to allow the input of their clinical, epidemiological and biological data in the cohort's database. Informed consent covers the use of clinical care data for research purposes, the record linkage of patient data with other databases and permission to collect patient biosamples for cell and pharmacogenomics analysis. The primary outcome 
of the cohort is to evaluate the virological efficacy and safety of DTG in both treatment-naive and treatmentexperienced patients. The secondary outcomes include the assessment of tolerability, changes in immunological, metabolic and inflammatory profile. Main statistical analyses are as follows: parametric and non-parametric test, as appropriate, to compare continuous and categorical values, survival analysis (ie, Kaplan-Meier estimator) and regression analyses (Cox regression, linear and logistic, as appropriate). As of 31 December 2018, 4139 patients have been enrolled in the cohort, prevalently male $(72.5 \%)$, with a median age of 50.4 years (IQR 42.2-55.6). Full patients' characteristics are shown in table 1. Median follow-up time was 19.7 months (IQR 10.7-28.7), with a cumulative time of follow-up of 6937 patient-years of follow-up (PYFU). Almost $90 \%$ of the enrolled naive patients were on an ARV regimen made up of a 2-NRTI backbone plus DTG (39.3\% with abacavir $(\mathrm{ABC})+3 \mathrm{TC}$ and $50.9 \%$ with emtricitabine (FTC) plus either tenofovir disoproxil fumarate (TDF) or tenofovir alafenamide (TAF)); conversely, less than two-thirds of the experienced subjects were on such standard regimens $(41.8 \%$ with ABC+3 TC and $16.9 \%$ with FTC plus TDF or TAF), while $16.6 \%$ switched to $3 \mathrm{TC}+\mathrm{DTG}, 10.1 \%$ switched to a dual therapy with a PI plus DTG and $7.1 \%$ to RPV+DTG. Table 2 comprehends all the analysed ARV regimens. Interestingly, there is a small group of 25 treatment -naïve patients on a dual regimen in our cohort; in this group, during 43.7 PYFU we observed just one virological failure (VF, defined as failure to achieve HIV-1 RNA below 50 copies $/ \mathrm{mL}$ after 6 months since ARV initiation) and four treatment discontinuations (TDs). The single patient who experienced VF was a 38-year-old woman starting DTG plus etravirine, presenting at 6 months an HIV viral load of 280000 copies $/ \mathrm{mL}$; she was then switched to FTC/TDF+DTG+DRV/r, achieving virological suppression. These data are of particular interest since data on safety and tolerability of two-drug regimen in treatment-naïve patients are only recently being published with the 48-week results of the GEMINI trials. ${ }^{23}$

Each patient usually attends one of the centres for a routine check-up every 3-4 months or when a new clinical event occurs, a new treatment is prescribed or a change in biological markers is observed. The overall 3 years probability of maintaining the prescribed regimen was $57.0 \%$. It is to be noted however that we considered as treatment interruption also the change of backbone while maintaining DTG or the optimisation to single tablet regimen (ie, from $\mathrm{ABC} / 3 \mathrm{TC}+\mathrm{DTG}$ to $\mathrm{ABC} / 3 \mathrm{TC} /$ DTG or from FTC/TDF to FTC/TAF). Discontinuation of the prescribed regimen was due to:

- Treatment simplification $(41.9 \%)$.

- Toxicities (29.7\%): gastrointestinal (GI) tract (11.7\%), neuropsychiatric $(11.0 \%)$, renal $(2.3 \%)$, other toxicities $(1.6 \%)$, hypersensitivity reaction $(3.1 \%)$.

- Virological failure $(4.3 \%)$.

- Treatment intensification (1.2\%).
- Drug-drug interaction (1.2\%).

- Other reasons (21.6\%): concern of cardiovascular disease $(2.2 \%)$, death $(1.4 \%)$, unspecified/unknown $(18.0 \%)$.

There were significant differences in the causes of discontinuation between naïve and experienced patients, with almost two-thirds of naïve patients $(64.6 \%)$ interrupting for proactive switch compared with the $36.4 \%$ of experienced patients. It is to be noted that in the last 2 years, both a single tablet regimen with $\mathrm{ABC} / 3 \mathrm{TC} / \mathrm{DTG}$ and a coformulation of FTC with TAF (instead of TDF) became widely available in Italy, prompting clinicians to further simplify ARV regimens.

Variables collected at patient enrolment include ages, gender, country of origin, mode of HIV transmission, date of primary infection if available and first positive HIV test. At baseline and at each follow-up visit, the following information are collected: body weight, viroimmunological markers of HIV infection such as CD4+ cell count, CD4/CD8 ratio and plasma HIV-1 RNA level, complete blood cell counts, lipid and liver enzymes; creatinine value and estimated glomerular filtration rate, antiretroviral drug start and stop dates, and the reasons for changing drug regimens, prophylaxis of opportunistic infections and comedications, serological and virological data on coinfection with hepatitis $\mathrm{C}$ virus and hepatitis V virus; date and type of AIDS and non-AIDS events (including cardiovascular events, cancers, neuropsychiatric events, bacterial infections and so on), changes in bone mineral density (dual-energy X-ray absorptiometry (DEXA) scan), date and cause of death (if applicable). In this regard, we recently published a work analysing trends of hospitalisation in HIV-infected patients. ${ }^{24}$ During 6937 PYFU, we observed 363 virological failures (described as two-consecutive HIV-1 RNA determination $>50$ copies $/ \mathrm{mL}$ or a single HIV-1 RNA $>1000$ copies $/ \mathrm{mL}$ in patients who previously achieved virological suppression or as failure to achieve HIV-1 RNA below 50 copies/ $\mathrm{mL}$ after 6 months since ARV initiation in treatmentnaïve patients), with an overall incidence of $5.2 \mathrm{VF}$ per 100 PYFU.

Currently, the ODOACRE cohort aims at collaborating with other European cohorts and databases, collecting data on HIV resistance mutations in order to further investigate DTG sensibility and efficacy in the context of resistance-associated mutations (RAMs). Preliminary collaboration with the 'Euresist cohort' led to a recent work showing that genotypic susceptibility score was a predictor of virological failure in INI-based regimens. ${ }^{25}$ Future projects also included the collection of biological samples to study HIV-DNA, inflammation markers (such as sCD14, interleukin 6, annexins and so on).

\section{Patient and public involvement}

The patients had no role in the design, recruitment and conduct of the study. 
Table 1 Patients' characteristics at last available update (31 December 2018)

\begin{tabular}{|c|c|c|c|c|}
\hline Variables & Overall $n=4139(\%)$ & $\begin{array}{l}\text { Treatment- } \\
\text { experienced } n=3658 \\
(\%)\end{array}$ & Naive $\mathrm{n}=\mathbf{4 8 1}(\%)$ & $P$ values \\
\hline Male, n (\%) & $2979(72.5)$ & $2609(71.7)$ & $370(78.7)$ & 0.001 \\
\hline Age (years), median (IQR) & $50.4(42.2-55.6)$ & $51.0(43.8-56.0)$ & $41.3(33.2-50.4)$ & $<0.001$ \\
\hline Risk factor, n (\%) & & & & $<0.001$ \\
\hline MSM & $1540(37.9)$ & $1310(36.4)$ & $230(50.1)$ & \\
\hline Eterosexual & $1572(38.7)$ & 1416 (39.3) & $156(34.0)$ & \\
\hline IDU & $753(18.5)$ & $712(19.8)$ & $41(9.0)$ & \\
\hline Other/Unknown & $196(4.9)$ & $164(4.6)$ & $32(6.9)$ & \\
\hline $\mathrm{HCV}^{\mathrm{C}}$ Ab positive, $\mathrm{n}(\%)$ & $833(21.2)$ & $804(22.8)$ & $29(7.3)$ & $<0.001$ \\
\hline HBsAg positive, n (\%) & $107(2.7)$ & $96(2.7)$ & $11(2.7)$ & ns \\
\hline CDC stage C, n (\%) & $837(26.9)$ & $774(28.0)$ & $63(17.8)$ & $<0.001$ \\
\hline Years from HIV diagnosis, median (IQR) & & $14.0(5.4-23.1)$ & l & / \\
\hline $\begin{array}{l}\text { Zenith HIV-RNA ( } \log _{10} \text { copies/mL), median } \\
\text { (IQR) }\end{array}$ & $4.82(4.29-5.37)$ & $4.82(4.29-5.36)$ & $5.05(4.57-5.56)$ & $<0.001$ \\
\hline Nadir CD4+ (cells/mmc), median (IQR) & $194.0(61.0-324.0)$ & $191.0(58.0-312.0)$ & $272.0(94.5-488.5)$ & $<0.001$ \\
\hline BL CD4+ (cell/mmc), median (IQR) & $583(365-810)$ & $601(400-823)$ & $330(110-560)$ & $<0.001$ \\
\hline Years on cART, median (IQR) & & $10.6(4.0-18.5)$ & l & l \\
\hline $\begin{array}{l}\text { Time on virological suppression (months), } \\
\text { median (IQR) }\end{array}$ & & $43.9(8.4-97.0)$ & / & / \\
\hline $\begin{array}{l}\text { Virologically suppressed patients at baseline, } \\
\mathrm{n}(\%)\end{array}$ & & $2222(80.4)$ & / & / \\
\hline Previous virological failure, $\mathrm{n}(\%)$ & & $1183(44.9)$ & / & / \\
\hline Therapies before switch, n (\%) & & & & / \\
\hline 2NRTI+PI & & $944(25.9)$ & & \\
\hline 2NRTI+INI & & 835 (22.9) & & \\
\hline 2NRTI+NNRTI & & $702(19.2)$ & / & \\
\hline Mono/Dual & & $763(20.9)$ & & \\
\hline Others & & $405(11.1)$ & & \\
\hline $\begin{array}{l}\text { Reasons for previous treatment } \\
\text { discontinuation, } \mathrm{n}(\%)\end{array}$ & & & & / \\
\hline Virological failure & & $209(5.7)$ & & \\
\hline Treatment Intensification & & $106(2.9)$ & & \\
\hline Dyslipidaemia & & $247(6.8)$ & / & \\
\hline Proactive switch/Simplification & & $1773(48.5)$ & & \\
\hline Gl toxicity & & $170(4.6)$ & & \\
\hline Renal toxicity & & $113(3.1)$ & & \\
\hline Neurological toxicity & & $34(0.9)$ & & \\
\hline Osteopoenia/Osteoporisis & & $61(1.7)$ & & \\
\hline Other toxicity & & $44(1.2)$ & & \\
\hline Hypersensitivity & & $20(0.5)$ & & \\
\hline Drug-drug interactions & & $155(4.2)$ & & \\
\hline Other/Unknown & & $723(19.8)$ & & \\
\hline
\end{tabular}

GI, gastrointestinal; INI, integrase inhibitor; NNRTI, non-nucleoside reverse transcriptase inhibitor ; NRTI, nucleoside reverse transcriptase inhibitor ; PI, protease inhibitor. 
Table 2 ARV regimens in the cohort

\begin{tabular}{|c|c|c|c|}
\hline ARV regimen & Overall & Treatment-experienced patients & Naïve patients \\
\hline $\mathrm{DTG}+\mathrm{ABC} / 3 \mathrm{TC}$ & $1718(41.5)$ & $1529(41.8)$ & $189(39.3)$ \\
\hline DTG+FTC/Tenofovir (either TDF or TAF) & $863(20.9)$ & $618(16.9)$ & $245(50.9)$ \\
\hline $\mathrm{DTG}+\mathrm{RPV}$ & $263(6.4)$ & $259(7.1)$ & $4(0.8)$ \\
\hline DTG+PI (boosted or unboosted) & $380(9.2)$ & $370(10.1)$ & $10(2.1)$ \\
\hline Other DTG-based dual regimen & $22(0.5)$ & $19(0.5)$ & $3(0.6)$ \\
\hline Other DTG-based regimen (three or more drugs) & $269(6.5)$ & $247(6.8)$ & $22(4.6)$ \\
\hline
\end{tabular}

ARV, antiretroviral; DTG, dolutegravir; PI, protease inhibitor; RPV, rilpivirine; TAF, tenofovir alafenamide ; 3TC, lamivudine; TDF, tenofovir disoproxil fumarate.

\section{Findings to date}

In recent years, various works by the ODOACRE cohort have been produced and published in full paper articles. The cohort's production has been mainly focused on the study of the effectiveness and tolerability of DTGbased ARV regimens. In 2017, we published a work on the safety of a switch strategy with DTG plus a dual-NRTI backbone, ${ }^{26}$ following reports of high rates of discontinuations of DTG-based regimens due to neuropsychiatric symptoms. In our work, we observed no virological failures during 1090 patient-months of follow-up and a $9.2 \%$ rate of TD, with a median time to TD of 81 days.

Baldin et $a l^{27}$ compared a switch regimen with FTC/ TDF plus DTG with a single tablet regimen with EVG and found that the 48-week estimated probability of continuing treatment was $74.5 \%$ for patients in the DTG group and $84.7 \%$ for those in the EVG group, and the difference was not statistically significant. Most recent works, meanwhile, mainly focus on the efficacy and safety of less drug regimens as switch strategies in virologically suppressed HIV-1-positive patients, since clinician's attention has shifted towards the analysis of risk/benefit ratio of dual therapies. Capetti $e t a t^{28}$ have described the effectiveness and tolerability of a dual therapy with RPV and DTG, with a median follow-up time of 101 weeks; in this study, the probability of maintaining virological suppression was $96.3 \%$ at week 96 while also observing a significant reduction of the low-density lipoprotein cholesterol/ high-density lipoprotein cholesterol ratio. Similarly, a work by Borghetti et $a l^{29}$ showed the results of our experience with a switch regimen of 3TC plus DTG in clinical practice; in this study, over 216.5 PYFU, we observed five virological failures with a probability of maintaining suppression of $95.1 \%$ at week 96. Finally, a comparison between the switch regimens of RPV+DTG and 3TC+DTG was published in $2018^{30}$; in this work, we observed no differences in the rate of virological failure between the two groups with no mutations to INI and with just one patient who developed a resistance mutation to NNRTIs at failure in the RPV group.
Rescue regimen in highly treatment-experienced patients

Our experience with highly treatment-experienced patients was well described in a work by Capetti et $a l,{ }^{31}$ showing the results of a rescue regimen of boosted darunavir plus DTG on 130 patients; about $90 \%$ of them had documented resistance to one to five ARV classes. After 48 weeks of follow-up, we observed a decrease in the proportion of patients harbouring active HIV replication from $40 \%$ at baseline to $6.1 \%$. Patients starting boosted DRV+DTG with HIV-RNA over 50 copies/mL presented a success rate of $88.5 \%$; meanwhile, $97.4 \%$ of those who started this regimen with HIV-RNA below 50 copies $/ \mathrm{mL}$ maintained virological suppression.

\section{DTG in naïve patient}

Following the results from the GEMINI trials, ${ }^{23}$ the use of a dual therapy with 3TC plus DTG in treatmentnaïve patients will become more common in clinical practice and we expect to describe the feasibility of this strategy in our centres in the short future. With these data obviously still months away, our experience in naïve patients is represented by two recent studies: Rossetti et $a l^{22}$ described the efficacy of DTG-based regimens (vs non-DTG-containing regimens) in achieving virological suppression in advanced HIV-infected naïve patients. Thirty-six per cent of patients in the DTG group achieved HIV-RNA below 50 copies/mL after 1 month of therapy (vs $17 \%$ of the non-DTG group) and $91 \%$ of them achieving suppression at 24 months (vs 89\%), while the frequency of IRIS was similar between groups. Another study by Lagi $e t a l^{33}$ compared the tolerability and viroimmunologic efficacy of DTG-based regimens versus other regimens in naïve patients with acute HIV infection, with both groups showing significant increase in CD4+ cell count and $\mathrm{CD} 4+/ \mathrm{CD} 8+$ ratio at 3, 6, 12, 24 and 36 months without significant differences between them.

\section{Further study plans}

Given the large data set available, the ODOACRE cohort aims at continuing its observational activity on DTGbased regimens, highlighting rates and reasons of DTG 
discontinuation for both standard three-drug regimen and dual regimen, with a focus also on ARV-naïve patients. The already published data, confirming the favourable metabolic profile of DTG, will be implemented with the assessment of inflammation and immunological status. Moreover, given the expanding interest in weight gain during INI-based regimens, ${ }^{16} 34$ the cohort will attempt to thoroughly study this matter in its large population. The collaboration with other cohorts, mainly collecting data on HIV genotypic test, will lead to investigate the role of RAMs on the virological outcome of DTG-containing regimens, also considering not only HIV-RNA but also HIV-DNA. Additional assessments will also be conducted on the adverse events reported during therapy with DTG in order to examine possible predictors of these events (ie, neuropsychiatric events).

\section{Strengths and limitations}

The main strength of the ODOACRE cohort is that it enrols all the patients starting a DTG-based regimen in each of the participating centres, with a grand total of 4139 patients as of December 2018, providing a real clinical practice scenario of the use of DTG in Italy. Another strength is the cumulative length of follow-up (6937 PYFU), which, along with the clinical, laboratory and demographic data of such a large number of patients, allows us to thoroughly define the safety and tolerability of the regimens. Our observational data on the dual therapy with RPV and DTG have anticipated the results of the SWORD trials; similarly, while the TANGO trials on the efficacy and tolerability of 3TC+DTG are still under way, ${ }^{35}$ our group has already described in multiple works the real-life feasibility of the said regimen.

A predominant feature of our cohort is that it is prevalently composed of highly treatment-experienced patients, with an overall median time from HIV diagnosis of 15.6 years (IQR 7.2-23.9), a median time on ARV of 11.4 years (IQR 5.0-18.7) and a median time of virological suppression of 3.9 years (IQR 0.8-8.2). Moreover, 44.9\% of the patients experienced at least one virological failure in their clinical history and $21.2 \%$ of them switched to DTG coming from a dual/mono regimen.

The main weakness of our study is that it only collects routine clinical data and therefore some data could be missing in the analysis. The observational nature of the study is prone to bias due to unmeasured confounders that may impact on the study endpoints. Inaccurate or incomplete reporting on non-AIDS-related events, concomitant medications and reasons for DTG discontinuation cannot be excluded. Furthermore, as in several other observational studies, patients who refused to give their consent to data collection may differ systematically on some of the baseline characteristics from patients who participated.

Moreover, although a yearly update is planned, a delay exists between local data collection and availability of an updated central database.

\section{COLLABORATION}

Data can be obtained on reasonable request. We encourage other clinical centres, even from other countries, to collaborate with our cohort. In particular, we aim to further investigate DTG-based regimens in several clinical contests and we would like to evaluate these regimens in comparison with other INIs and other ARV drugs in order to try to answer the many questions that still come from clinical practice.

\section{Author affiliations}

${ }^{1}$ Università Cattolica del Sacro Cuore, Facoltà di Medicina e Chirurgia, Roma, Italy ${ }^{2}$ Università degli Studi di Milano Dipartimento di Scienze Cliniche Luigi Sacco, Milano, Lombardia, Italy

${ }^{3}$ Università degli Studi di Modena e Reggio Emilia Facoltà di Medicina e Chirurgia, Modena, Emilia-Romagna, Italy

${ }^{4}$ Division of Tropical and Infectious Diseases, Azienda Ospedaliera Careggi, Firenze, Italy

${ }^{5}$ Infectious Dermatology and Allergology Unit, IF0, Roma, Lazio, Italy

${ }^{6}$ Department of Clinical, Surgical and Experimental Sciences, Sassari University Hospital, Sassari, Sardegna, Italy

${ }^{7}$ Department of Public Health and Infectious Diseases, Universita degli Studi di Roma La Sapienza, Roma, Lazio, Italy

${ }^{8}$ Infectious Disease Clinic- Department of Medicine and Science of Aging, School of Medicine, "G. d'Annunzio" University, Chieti, Italy

${ }^{9}$ Università degli Studi di Siena Facoltà di Medicina e Chirurgia, Siena, Toscana, Italy ${ }^{10}$ Università Politecnica delle Marche Facoltà di Medicina e Chirurgia, Ancona, Marche, Italy

${ }^{11}$ UOC Malattie Infettive, Fondazione Policlinico Universitario Agostino Gemelli IRCCS, Roma, Italy

\section{Twitter Maria Vittoria Cossu @maria.cossu}

Acknowledgements First at all, the authors would like to thank all patients enrolled in the cohort. We also thank all clinical centres that participated in the ODOACRE cohort and in particular A. Emiliozzi, D. Moschese, C. Picarelli, F. Lombardi, S. Belmonti, S. Lamonica, V. Delle Donne, D. Farinacci, G. d'Ettorre, C. M. Mastroianni (Rome), T. Formenti (Milan), A. De Vito (Sassari), J. Vecchiet (Chieti) and A. Giacometti (Ancona).

Contributors ACic, GB, LC, FV, BR, AD, MVC, SRes, WG, FL, AG, MC, LB and $A B$ managed patients and collected data. $A C i c$ and $G B$ collaborated with the other clinical centers and wrote the manuscript. ACap, VB, GS, AL, GM, CM and SRus critically revised the manuscript. SDG conceived the work and revised the manuscript. All authors approved the submitted version of this manuscript.

Funding The cohort was supported by ViiV Healthcare Europe, which provided an unrestricted grant to the Infectious Disease Unit of the University of Siena.

Map disclaimer The depiction of boundaries on this map does not imply the expression of any opinion whatsoever on the part of BMJ (or any member of its group) concerning the legal status of any country, territory, jurisdiction or area or of its authorities. This map is provided without any warranty of any kind, either express or implied.

Competing interests $\mathrm{GB}$ received travel grant from Gilead. ACa has received a personal grant from AB, Gilead and ViiV. GS has received funds for speaking by Gilead, Merk, Janssen, Abbvie, ViiV. AL received personal fees from BMS, Gilead, Merck, ViiV, AbbVie and Janssen and grants from BMS, Gilead, ViiV and Janssen. GM is in an ongoing relation as board member for ViiV Healthcare, Gilead Sciences and Jannsen. BR received travel grants from Jannsen, ViiV, Gilead MSD and received grants for consultancy from Abbvie, MSD, Viiv. AG received speaker fees from Mylan. AB has received non-financial support from Bristol-Myers Squibb and ViiV Healthcare, and personal fees from Gilead Sciences. CM has participated in advisory boards, received study grants and/or speaker honoraria from Abbvie, Gilead, Viiv, Janssen, Angelini, BMS and MSD. SR received research grants to his Institution from ViiV Heathcare, Gilead Sciences and Jannsen, outside the submitted work; he was also a paid consultant for ViiV Heathcare, Gilead Sciences, Merck Sharp and Dohme, Bristol-Myers Squibb, Janssen and Mylan. SDG was a paid consultant or member of advisory boards for Gilead, ViiV Healthcare, Janssen-Cilag, Merck Sharp \& Dohme and Bristol-Myers Squibb. 
Patient consent for publication Not required.

Ethics approval The study was approved by local ethical committees (protocol number of the promoter centre: 5284/15).

Provenance and peer review Not commissioned; externally peer reviewed.

Data availability statement All data relevant to the study are included in the article or uploaded as supplementary information.

Open access This is an open access article distributed in accordance with the Creative Commons Attribution Non Commercial (CC BY-NC 4.0) license, which permits others to distribute, remix, adapt, build upon this work non-commercially, and license their derivative works on different terms, provided the original work is properly cited, appropriate credit is given, any changes made indicated, and the use is non-commercial. See: http://creativecommons.org/licenses/by-nc/4.0/.

\section{ORCID iDs}

Gianmaria Baldin http://orcid.org/0000-0003-4623-9976

Andrea Giacomelli http://orcid.org/0000-0003-3685-4289

Stefano Rusconi http://orcid.org/0000-0002-0375-9990

\section{REFERENCES}

1 Palella FJ, Delaney KM, Moorman AC, et al. Declining morbidity and mortality among patients with advanced human immunodeficiency virus infection. HIV outpatient study Investigators. N Engl J Med 1998;338:853-60.

2 Maggi P, Di Biagio A, Rusconi S, et al. Cardiovascular risk and dyslipidemia among persons living with HIV: a review. BMC Infect Dis 2017; $17: 551$.

3 Brown TT, Qaqish RB. Antiretroviral therapy and the prevalence of osteopenia and osteoporosis: a meta-analytic review. AIDS 2006;20:2165-74.

4 Wong E, Trustman N, Yalong A. Hiv pharmacotherapy: a review of integrase inhibitors. JAAPA 2016;29:36-40.

5 Cahn P, Pozniak AL, Mingrone H, et al. Dolutegravir versus raltegravir in antiretroviral-experienced, integrase-inhibitor-naive adults with HIV: week 48 results from the randomised, double-blind, noninferiority SAILING study. Lancet 2013;382:700-8.

6 Raffi F, Jaeger H, Quiros-Roldan E, et al. Once-Daily dolutegravir versus twice-daily raltegravir in antiretroviral-naive adults with HIV-1 infection (SPRING-2 study): 96 week results from a randomised, double-blind, non-inferiority trial. Lancet Infect Dis 2013;13:927-35.

7 Pappa K, Baumgarten A, Felizarta F, et al. Once daily dolutegravir + abacavir/lamivudine is superior to efavirenz/tenofovir/emtricitabine in treatment-naive HIV subjects: 144-Week Results - SINGLE (ING114467). Washington, DC: 54th Interscience Conference on Antimicrobial Agents and Chemotherapy, 2014.

8 Eron JJ, Clotet B, Durant J, et al. Safety and efficacy of dolutegravir in treatment-experienced subjects with raltegravir-resistant HIV type 1 infection: 24-week results of the Viking study. J Infect Dis 2013;207:740-8

9 Castagna A, Maggiolo F, Penco G, et al. Dolutegravir in AntiretroviralExperienced patients with Raltegravir- and/or Elvitegravir-Resistant HIV-1: 24-week results of the phase III VIKING-3 study. J Infect Dis 2014;210:354-62.

10 Vavro G, Huang J, Avatapally C, et al. Durable efficacy and limited integrase resistance in subjects receiving dolutegravir after failing a prior INI regimen: week 48 results from VIKING-3 (Abstract O_10). Barcelona, Spain: 12th European Workshop on HIV \& Hepatitis, 2014.

11 Rossetti B, Montagnani F, De Luca A. Current and emerging two-drug approaches for HIV-1 therapy in ART-naïve and ARTexperienced, virologically suppressed patients. Expert Opin Pharmacother 2018;19:713-38.

12 DHHS. Guidelines for the use of antiretroviral agents in adults and adolescents living with HIV. Department of Health and Human Services, 2017

13 Hoffmann C, Welz T, Sabranski M, et al. Higher rates of neuropsychiatric adverse events leading to dolutegravir discontinuation in women and older patients. HIV Med 2017;18:56-63.

14 de Boer MGJ, van den Berk GEL, van Holten N, et al. Intolerance of dolutegravir-containing combination antiretroviral therapy regimens in real-life clinical practice. AIDS 2016;30:2831-4.

15 Zash R, Makhema J, Shapiro RL. Neural-Tube defects with dolutegravir treatment from the time of conception. N Engl J Med 2018;379:979-81.
16 Bourgi K, Rebeiro PF, Turner M, et al. Greater weight gain in treatment naïve persons starting Dolutegravir-Based antiretroviral therapy. Clin Infect Dis 2019:ciz407.

17 Maggi P, Montinaro V, Rusconi S, et al. The problem of renal function monitoring in patients treated with the novel antiretroviral drugs. HIV Clin Trials 2014;15:87-91.

18 Reese MJ, Savina PM, Generaux GT, et al. In vitro investigations into the roles of drug transporters and metabolizing enzymes in the disposition and drug interactions of dolutegravir, a HIV integrase inhibitor. Drug Metab Dispos 2013;41:353-61.

19 Linee Guida Italiane sull'utilizzo della Terapia Antiretrovirale e la gestione diagnostico-clinica delle persone con infezione da HIV-1 Edizione 2017.

20 Llibre JM, Hung C-C, Brinson C, et al. Efficacy, safety, and tolerability of dolutegravir-rilpivirine for the maintenance of virological suppression in adults with HIV-1: phase 3 , randomised, non-inferiority SWORD-1 and SWORD-2 studies. Lancet 2018;391:839-49.

21 Boswell R, Foisy MM, Hughes CA. Dolutegravir dual therapy as maintenance treatment in HIV-infected patients: a review. Ann Pharmacother 2018;52:681-9.

22 Joly V, Burdet C, Landman R, et al. Dolutegravir and lamivudine maintenance therapy in HIV-1 virologically suppressed patients: results of the ANRS 167 trial (LAMIDOL). J Antimicrob Chemother 2018.

23 Cahn P, Madero JS, Arribas JR, et al. Dolutegravir plus lamivudine versus dolutegravir plus tenofovir disoproxil fumarate and emtricitabine in antiretroviral-naive adults with HIV-1 infection (GEMINI-1 and GEMINI-2): week 48 results from two multicentre, double-blind, randomised, non-inferiority, phase 3 trials. Lancet 2018.

24 Bellino S, Borghetti A, Lombardi F, et al. Trends of hospitalisations rates in a cohort of HIV-infected persons followed in an Italian hospital from 1998 to 2016. Epidemiol Infect 2019;147:e89.

25 Borgheti A, Ciccullo A, Lombardi F, et al. Gss of NRTI-BACKBONE predicts time to virological failure of INI-BASED regimens. Abstract $547,26^{\circ}$ conference on retroviruses and opportunistic infections (CROI), Seattle, USA 2019.

26 Borghetti A, Baldin G, Capetti A, et al. Efficacy and tolerability of dolutegravir and two nucleos(t)ide reverse transcriptase inhibitors in HIV-1-positive, virologically suppressed patients. AIDS 2017;31:457-9.

27 Baldin G, Ciccullo A, Capetti A, et al. Efficacy and safety of switching to dolutegravir plus emtricitabine/tenofovir disoproxil fumarate (TDF) or elvitegravir/cobicistat/emtricitabine/TDF in virologically suppressed HIV-infected patients in clinical practice: results from a multicentre, observational study. HIV Med 2018.

28 Capetti AF, Cossu MV, Sterrantino G, et al. Dolutegravir plus rilpivirine as a switch option in CART-Experienced patients: 96-Week data. Ann Pharmacother 2018;52:740-6.

29 Borghetti A, Baldin G, Lombardi F, et al. Efficacy and tolerability of lamivudine plus dolutegravir as a switch strategy in a multicentre cohort of patients with suppressed HIV-1 replication. HIV Med 2018.

30 Ciccullo A, Baldin G, Capetti A, et al. A comparison between two dolutegravir-based two-drug regimens as switch strategies in a multicentre cohort of HIV-1-infected patients. Antivir Ther 2018.

31 Capetti AF, Cossu MV, Orofino G, et al. A dual regimen of ritonavir/darunavir plus dolutegravir for rescue or simplification of rescue therapy: 48 weeks' observational data. BMC Infect Dis 2017; $17: 658$

32 Rossetti B, Baldin G, Sterrantino G, et al. Efficacy and safety of Dolutegravir-Based regimens in advanced HIV-infected naïve patients: results from a multicenter cohort study. Abstract supplement HIV Glasgow 2018. Journal of the International AIDS Society 2018:21:e25187.

33 Lagi F, Baldin G, Colafigli M, et al. Viro-immunological efficacy and tolerability of dolutegravir-based regimens compared to regimens based on other integrase strand inhibitors, protease inhibitors or non-nucleoside reverse transcriptase inhibitors in patients with acute HIV-1 infection: a multicenter retrospective cohort study. Int $J$ Antimicrob Agents 2019;54:487-90.

34 Menard A, Meddeb L, Tissot-Dupont $\mathrm{H}$, et al. Dolutegravir and weight gain: an unexpected bothering side effect? AIDS 2017:31:1499-500.

35 ClinicalTrial.gov. Switch study to evaluate dolutegravir plus lamivudine in virologically suppressed human immunodeficiency virus type 1 positive adults (tango). ClinicalTrials.gov identifier NCT03446573. Available: https://clinicaltrials.gov/ct2/show/ NCT03446573 